Résumés des conférences et travaux

\title{
Linguistique berbère
}

\section{Amina Mettouchi}

\section{OpenEdition \\ Journals}

\section{Édition électronique}

URL : https://journals.openedition.org/ashp/1111

DOI : 10.4000/ashp.1111

ISSN : 1969-6310

Éditeur

Publications de l'École Pratique des Hautes Études

\section{Édition imprimée}

Date de publication : 1 octobre 2011

Pagination : $43-46$

ISSN : 0766-0677

\section{Référence électronique}

Amina Mettouchi, «Linguistique berbère ", Annuaire de l'École pratique des hautes études (EPHE), Section des sciences historiques et philologiques [En ligne], 142 | 2011, mis en ligne le 18 juillet 2011, consulté le 06 juillet 2021. URL : http://journals.openedition.org/ashp/1111; DOl : https://doi.org/10.4000/ashp. 1111 


\title{
LINGUISTIQUE BERBÈRE
}

\author{
Directrice d'études : $\mathrm{M}^{\mathrm{me}}$ Amina Mettouchi
}

Programme de l'année 2009-2010 : I. La référence en berbère. - II. L'opposition en berbère.

Dans la mesure où l'hypothèse la plus communément partagée par les berbérisants est celle d'une origine démonstrative des marques d'état (cf. conférence II), il nous est apparu opportun de commencer l'année par la question de la référence, et d'organiser la réflexion autour des démonstratifs et de la détermination nominale.

Les textes analysés étant presque tous des contes (à part quelques textes ethnographiques touaregs, et de Ghadamès), nos conclusions restent limitées à ce genre pour l'instant.

Outre nos auditeurs, doctorants locuteurs de tamazight et de kabyle, nous avons eu le plaisir d'accueillir très régulièrement Lionel Galand, qui a bien voulu participer aux conférences et nous faire bénéficier de son immense savoir et sa grande finesse d'analyse.

\section{La référence en berbère}

Dans un premier temps nous avons étudié l'emploi du numéral «un ». Sachant qu'un nom en berbère peut apparaître sans déterminant, et avoir une interprétation définie ou indéfinie selon les contextes, la question se posait de savoir dans quel cas le numéral « un » était employé comme déterminant, de préférence à la forme nue du nom. Une étude de textes chleuhs (Stroomer 2003 et 2004) nous a permis de voir que l'emploi de ce déterminant, lorsqu'il n'est pas strictement numéral, revient à focaliser le référent, soit de manière contrastive (l'un et l'autre), soit pour indiquer qu'il s'agit d'un référent nouveau qu'il s'agit ensuite de commenter et de développer. Nous avons ainsi rejoint les conclusions de Paulette Galand-Pernet (1981, p. 25-26) qui considère yan en chleuh comme ayant une fonction relationnelle dans la structuration du conte. Le touareg de l'Ahaggar ne présente pas la même configuration d'emplois. D'abord le déterminant « un » peut précéder ou suivre le nom (cette dernière configuration est de loin la plus fréquente dans les textes étudiés (Foucault 1922). Ensuite, la dimension d'altérité est très présente, mais on ne trouve pas les emplois discursifs focalisants relevés en chleuh. En revanche, le déterminant « un » est souvent employé pour insister sur le caractère quelconque d'un référent qui pourrait sinon être interprété comme défini. En kabyle (Fichier de documentation berbère, 1967, et textes oraux transcrits par A. Mettouchi), l'emploi du déterminant « un » est plus rare qu'en chleuh, ou même qu'en touareg. On trouve quelques emplois où yiwen permet d'introduire de nouveaux protagonistes, mais la plupart du temps c'est la valeur numérale ou, plus rarement, indéfinie qui prévaut. On voit donc se dessiner, à travers ces trois variétés berbères, et dans des textes narratifs, les chemins d'une grammaticalisation plus ou moins avancée, et surtout, ayant privilégié différemment selon les langues, les traits 
contenus dans la notion numérale « un » (opposition d'altérité, ou rapport de l'un à l'ensemble, focalisation, indéfinitude...).

Nous sommes alors passés à l'étude des démonstratifs. Nous avons analysé le système du chleuh, décrit dans Galand (1988, p. 219), celui du kabyle, (Mettouchi à paraître), celui du touareg de l'Ahaggar (Prasse 2005), et celui du berbère de Ghadamès (Lanfry 1968). Les systèmes représentés dans les textes narratifs ou ethnographiques sont simplifiés par rapport à ceux employés en conversation (cf. Mettouchi à paraître). Après avoir étudié rapidement les emplois des démonstratifs proximal et distal nous nous sommes concentrés sur les contextes d'emploi dits « de reprise », en particulier en ghadamsi et en kabyle. Loin de correspondre uniquement à des anaphores, ces emplois sont complexes et diversifiés, et participent davantage de la dimension pragmatique que de la dimension référentielle (Mettouchi à paraître) : le démonstratif « de reprise », que l'on peut associer aux traits « nouveau dans le discours » et « ancien pour l'interlocuteur » (« discourse new » and « hearer old», cf. Diessel 1999) s'associe aux référents qui sont des topiques discursifs (au niveau de l'épisode). Cette conclusion approfondit les remarques de Galand-Pernet (1981: 26) sur le rôle de $L i$ en chleuh.

L'étude de la grammaticalisation des démonstratifs « de reprise » a donné des résultats intéressants: tandis qu'en chleuh l'évolution se fait du démonstratif vers le relateur (lli fluctuant entre la fonction de déterminant suffixe du nom antécédent et celle d'élément introducteur de la relative), en kabyle et en ghadamsi, cette voie de grammaticalisation recensée dans Heine et Kuteva (2004) n'est pas empruntée; c'est la voie de grammaticalisation vers le déterminant défini qui prédomine en ghadamsi. Pour autant, le processus n'est pas achevé dans cette variété, puisque malgré la fréquence importante d'emploi du démonstratif « de reprise »-i, il existe encore des occurrences de noms à valeur définie non suivis de cet élément. En tahaggart nous avons constaté que l'emploi des démonstratifs en fonction de déterminant est peu grammaticalisé, les emplois pronominaux étant très importants ( $w a \mathrm{n}+\mathrm{N} ; w a+$ relative).

Nous avons conclu de ce tour d'horizon que la dimension référentielle n'était pas dominante dans l'emploi des démonstratifs « de reprise » en berbère : aucun des éléments associés aux véritables « articles définis » des langues qui en possèdent (influence de la sémantique des noms: comptable/non-comptable, abstrait/concret, nom propre/commun, unicité du référent, etc.) ne sont des traits pertinents pour le choix de l'emploi de ces démonstratifs. Ce choix n'est pas sémantique, mais discursif.

Cette conférence sera approfondie en 2011-2012, dans le cadre plus général de la modalité.

\section{L'Opposition d'état en berbère}

La plupart des langues ou variétés berbères connaissent l'opposition d'état, qui distingue morphologiquement deux formes du nom, dont les emplois sont fonctionnellement divers, du moins en apparence: complément du nom vs adjectif, sujet vs objet après le verbe, complément de certaines prépositions $v s$ d'autres, quantification, topicalisation, etc. Cette opposition a fait couler beaucoup d'encre chez les berbérisants, depuis André Basset (1932) jusqu'à Lionel Galand (2010). L'objectif de cette conférence était de réexaminer les hypothèses concernant la fonction de cette opposition, à la lumière des textes. 
Nous avons commencé par analyser la morphologie de la distinction d'état, qui ne se présente pas de la même manière en chleuh ou en kabyle, d'une part, et en touareg d'autre part. Nous avons distingué le processus historique de dérivation - complexifié par les changements phonologiques qui ont donné lieu à des distinctions entre noms à voyelle constante et noms à voyelle non-constante - de la dérivation synchronique, telle que l'on peut la voir à l'œuvre dans les emprunts par exemple, lorsque ceux-ci distinguent l'état.

Nous avons analysé les emplois de l'état libre et de l'état d'annexion, et avons constaté que certaines fonctions, comme la distinction du sujet et de l'objet en position postverbale, n'étaient que rarement à l'œuvre en texte, du fait de la tendance des langues à n'exprimer sous forme nominale qu'un des arguments d'une prédication (l'autre étant en général pronominal). Lorsqu'il arrive que deux arguments postverbaux soient exprimés sous forme nominale, l'ordre relatif de ces éléments ( $\mathrm{S}$ avant $\mathrm{O})$ est très largement prédominant. Il s'agit là peut-être de stratégies compensatoires, sachant que l'opposition d'état n'est plus marquée que pour environ la moitié des noms en kabyle et en chleuh.

Nous avons examiné les diverses hypothèses avancées par les linguistes pour expliquer la fonction de cette opposition (on peut trouver le commentaire d'une partie d'entre elles dans Mettouchi 2008). Nous nous sommes arrêtés notamment à celle développée par König (2006), après Aikhenvald (1995), qui considère l'état d'annexion comme un cas nominatif marqué. Nous avons montré que cette interprétation casuelle ne se fait qu'au prix d'une importante distorsion du concept de nominatif, et que l'analyse néglige le domaine de la modification nominale dans son ensemble, pour ne s'intéresser qu'à celui des relations grammaticales.

En somme, nous avons pu constater que l'opposition d'état était bel et bien une opposition originale, qui ne peut se réduire à des fonctions relevées dans d'autres langues. Elle permet de distinguer un nom modifiant référentiellement le constituant qui précède (état d'annexion) d'un nom n'opérant pas de modification référentielle du constituant précédent (l'état libre) (Mettouchi et Frajzyngier, soumis). Cette distinction assez abstraite se réalise de manière très diversifiée en berbère.

\section{Textes cités}

Cinq contes kabyles, Fort-National (Algérie), Fichier de documentation berbère, 1967.

Foucault (C. DE) et Calassanti-Motilinsky (A. DE), Textes touaregs en prose, Aix-enProvence, Édisud, 1984 (1922).

LANFry (J.), Ghadamès. Étude linguistique et ethnographique, Fort-National (Algérie), Fichier de documentation berbere, 1968.

Stroomer (H.), Tashelhiyt Berber Texts from the Ayt Brayyim, Lakhsas and Guedmioua Region (South Morocco), Cologne, Rüdiger Köppe, 2003.

-, Tashelhiyt Berber Texts from Ida u Tanan (South Morocco), Cologne, Rüdiger Köppe, 2004.

\section{Ouvrages et articles cités}

Aikhenvald (Alexandra), « Split Ergativity in Berber languages », St.Petersburg Journal of African Studies, 4 (1995), p. 39-68. 
BAsSet (André), « Note sur l'état d'annexion en berbère », Bulletin de la Société de linguistique de Paris, 33/2 (1932), p. 173-174

Diessel (H.), Demonstratives: Form, Function and Grammaticalization, Amsterdam Philadelphie, John Benjamins, 1999.

Galand (Lionel), «Le berbère ", dans J. Perrot (éd.), Les Langues dans le monde ancien et moderne, $3^{\mathrm{e}}$ partie : Les Langues chamito-sémitiques (textes recueillis par D. Cohen), Paris, CNRS, 1988, p. 207-242.

—, Regards sur le berbère, Milan, Centro Studi Camito-Semitici, 2010.

Galand-Pernet (P.), «Signalisation sur la route du Conte. Esquisse d'un système sémiologique », Bulletin de littérature orale arabo-berbère, 12 (1981), p. 15-40.

Heine (B.) et Kuteva (T.), The World Lexicon of Grammaticalization, Cambridge, CUP, 2004.

KöNIG (Christa), « Marked nominative in Africa », Studies in Language, 30/4 (2006), p. 655732.

Metтouchi (A.), « Case-marking, Syntactic domains and Information structure in Kabyle (Berber) », dans Frajzyngier et Shay (éd.), Interaction of Syntax and Morphology: Case studies in Afroasiatic, Amsterdam - Philadelphie, John Benjamins, 2008 (TLS, 75), p. 7-40.

—, «Démonstratifs et construction de la référence en kabyle », dans A. Mettouchi (éd), Mélanges offerts à Paulette Galand-Pernet et Lionel Galand à l'occasion de leur $90^{\circ}$ anniversaire, Cologne, Rüdiger Köppe, à paraître.

Mettouchi (A.) et Frajzyngier ( Z.), « Prosodic Group and Grammatical relations: the Annexed and Absolute states in Kabyle », soumis.

Prasse (Karl-G.), Manuel de grammaire touarègue : Syntaxe, Schwülper, Cargo Verlag, 2005. 